\title{
Are Noble Metals the Ideal Material for Interfaces in Capillary GC?
}

\author{
K. Grob \\ GC-Laboratory, ETH Zürich, EAWAG, CH-8600 Dübendorf, Switzerland
}

\begin{abstract}
Summary
According to the experimental evidence reported in this paper, the inner surface of tubings of Pt and other noble metals may, under certain conditions, become strongly active. The activity mav increase to such an extent that severe band broadening, tailing, and even total disappearing of organic substances of various classes (not just polar ones) are caused. Typically, noble metals constantly adapt their surface to the actual ambient conditions. It is not possible to maintain their surface in a given, preselected state. The most significant variable parameter influencing the surface is the nature of the effluent from a GC column. Upon column interchange, drastic changes in the surface characteristics of noble metal tubings can be observed. In contrast, a glass surface can be permanently inactivated and is not affected by varying effluents when columns are interchanged.
\end{abstract}

The use of noble metal tubing to connect gas chromatographic columns to injectors and detectors, as well as to auxiliary parts such as splitting or back flushing valves is as old as gas chromatography itself. It found additional application when the design of $\mathrm{gc} / \mathrm{ms}$ interfaces began attracting increasing attention, and special refinements were introduced for capillary columns. The literature on these aspects has grown to such an extent that we do not even attempt to cite representative papers.

A substantial breakthrough occurred when Etzweiler, Neuner-Jehle, and Zarske $[1,2]$ added their technique of connecting metal tubings and glass capillaries without dead volume. This technique opened new horizons for the application of glass capillary columns. The possibilities of new applications appeared to be unlimited. In view of the latest results it appears as though the general acceptance may have been somewhat too uncritical.

In our laboratory, noble metal tubings were primarily used for $\mathrm{gc} / \mathrm{ms}$ coupling. Our interface was designed in a way to allow easy and rapid column interchange [3]. This followed from our principle of working out a given analy tical problem as far as possible by pure gc. For the subsequent identification work on the ms we wished to use the same gc column. Thus, a given column remained coupled no longer than half a day or a day.
Apart from the general success, this work produced some scattered and unintelligible failures: sometimes total ion monitored gas chromatograms from the same column looked much poorer than FID monitored ones. Comprehensive studies involving comparison of various noble metal tubings, as well as numerous variations in cleaning and deactivating their surface, did not provide the missing insight into the underlying properties and processes. More consistent information accumulated only when we started studying the problems with purely gas chromatographic means. It is the purpose of this paper to present the major results obtained in this way.

\section{Experimental}

The metal tubing under study was mounted between a glass capillary column and the FID. In most cases, the following sequence was used (see schematic presentation in Fig. 1). The inlet of the column was mounted in the injection port as usual, the outlet was connected to one end of the metal tubing by Teflon shrink tubing, the other end was connected, again with Teflon, to a $12 \mathrm{~cm}$ piece of glass capillary, which led to the flame tip of the FID.

For comparison, several modifications of this system were tried, namely, leaving out the final glass capillary by connecting the metal tubing directly to the flame tip; keeping the Teflon shrink tubing connections in a helium atmosphere to eliminate diffusion of oxygen; leaving out the metal tubing by connecting the column outlet to the final glass capillary piece or directly to the FID. To assimilate the flow resistance of a narrow bore metal capillary, a suitably selected glass restriction was inserted between column and FID. Thus, comparisons between systems with and without metal tubing were conducted under essentially identical pressure/flow conditions. Column and metal tubing were automatically kept at the same temperature.

We studied the influence of the following metal tubings; $40 \mathrm{~cm} \times 0.3 \mathrm{~mm}$ i.d. pieces of gold, gold-platinum $60-$ $40 \%$, platinum, platinum-iridium 90-10\%; $50 \mathrm{~cm} \times 0.15$ $\mathrm{mm}$ i.d. pieces of platinum and platinum-iridium.

The glass capillary columns used were $15-30 \mathrm{~m}$ long and $0.28-0.35 \mathrm{~mm}$ bore, coated with one of the following liquid phases, 0V-1, SE-52, 0S-124, Ucon HB 5100, Emulphor ON 870 (E), PEG 20'000, Silar 10 C.

Most tests were run with our usual adsorption/polarity test mixture, containing 5-nonanone, 1-octanol, naph talene, and three neighboring $n$-alcanes, dissolved in hexane. Carrier gases were hydrogen or helium. 


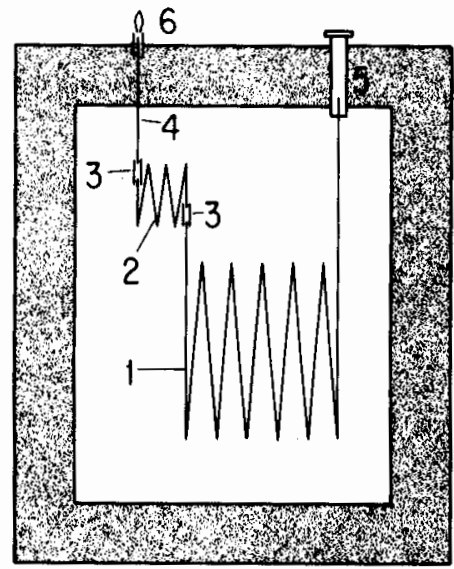

Fig. 1

- Schematic representation of lay-out. (1) glass capillary column, (2) coiled $50 \mathrm{~cm}$ noble metal capillary, (3) PTFE shrink tubing connections, (4) piece of inactivated glass capillary, (5) vaporizer, (6) FID.

Connecting glass capillaries were of soft glass, $0.3 \mathrm{~mm}$ bore. They were filled with $20 \% \mathrm{HCl}$, kept at $90^{\circ} \mathrm{C}$ for $10 \mathrm{~min}$, and inactivated by rinsing with a $0.5 \%$ solution of PEG $20 \mathrm{M}$ in methylene chloride. No heat treatment was applied, since this occurred automatically during use.

\section{Results}

The number of variable parameters in our study was far too large for carrying out a complete investigation within reasonable time. Originally we had assumed, for example, that there exist characteristic differences in the behaviour among the noble metals we studied. After observing, however, that these differences are relatively small, we dropped further differentiation. Thus we use the designation "Pt" in the following discussion for the entire range of noble metals under study. We assume, furthermore, that our observed effects depend on chromatographic conditions, such as temperature, flow rate, sample size, etc. Since it was hardly feasible, especially with phases of different polarity, to select and maintain standard conditions, we tried to avoid significant differences in conditions without attempting to elucidate the influence of single parameters. With these limitations in mind the following experimental observations may be noted.

1. We are not able to find any permanent or, at least, very long lasting inactivation of the Pt surface.

2. Using the Pt surface in the oxidized or reduced state (as produced by heating in the flame under oxygen or hydrogen flow respectively) did not influence the long term characteristics of the surface.

3. Oxidized and reduced, as well as surfaces treated otherwise (e.g. heated unter helium flow, washed with inorganic acids or bases, or with organic pure solvents) showed intense adsorption (1-octanol tailed badly or disappeared).
4. Inactivation with e.g. PEG solution, or application of surface modifiers such as ammonium salts (e.g. gas quat) or phosphonium salts (e.g. tetraphenylphosphonium chloride) produced transient inactivation only, disappearing after 2 hours at $200{ }^{\circ} \mathrm{C}$.

5. We did not observe any significant influence of carrier gas (helium or hydrogen).

6. No influence of oxygen traces, as could possibly penetrate through Teflon connections, was observed.

7. The Pt surface shows low activity only when a suitable inactivating agent is constantly supplied to it.

8. Qualitative selection of this agent seerns to be much more important than quantitative supply.

9. A very effective inactivating agent was found to be the carrier gas leaving a Ucon $H B 5100$ column, even when this column was perfectly conditioned and showed almost no bleeding.

10. The gases leaving all other columns we tested show a much weaker inactivating effect. It is, of course, possible that among the numerous phases used in GC but not tested in this study, substances equivalent or superior to Ucon HB 5100 may be found.

11. The poor inactivating effect of an unsuitable phase (e.g. PEG, most silicones, E), is not significantly increased when freshly coated columns with high bleeding are used.

12. The surface-modifying effects of most column effluents are reversible. For instance, the inactivating effect caused by a Ucon HB column is rapidly destroyed by the effluents of a PEG column, and may be restored by switching back to a Ucon HB column.

13. An example of irreversible activation: a Silar $10 \mathrm{C}$ column, connected to a Pt capillary for 3 days at $240^{\circ}$ produced a very high activity which could not be eliminated by prolonged coupling to a Ucon HB column, by rinsing with various solvents and aqueous reagents, or by glowing in an oxygen atmosphere.

14. The observed surface activity may primarily affect polar substances. When produced by different column effluents it may act specially on apolar substances such as alkanes. Thus, the activity seems to be based on more than one mechanism.

15. As a rule, aromatic hydrocarbons were always least affected by active Pt surfaces.

16. Ucon HB 5100 applied in liquid phase (dissolved) in methylene chloride) showed no more durability of inactivation than other liquid agents.

17. If Pt tubing is replaced by an inactivated glass capillary, practically permanent inactivity, independent of column coating, is observed.

\section{A typical example}

Out of a large number of similar observations we present here an arbitrarily selected example to elucidate the discussed effects. 


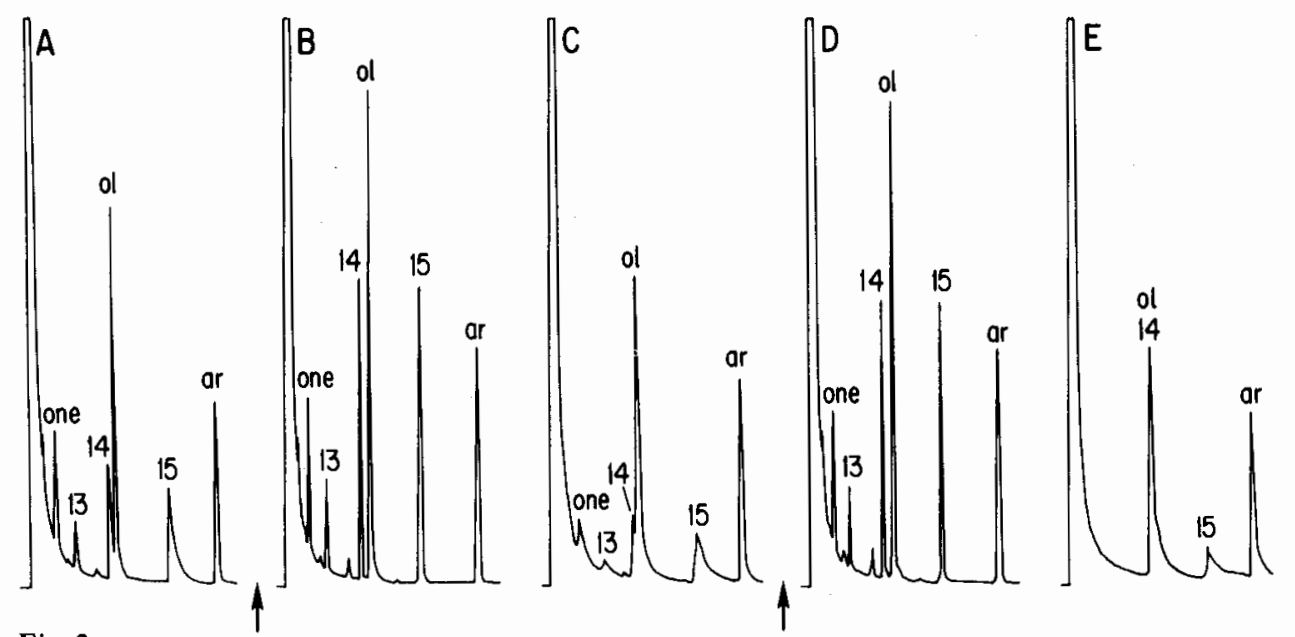

Fig. 2

Adsorption polarity test mixture eluted, through $50 \mathrm{~cm} \mathrm{Pt/Ir} \mathrm{capillary,} \mathrm{from} \mathrm{glass} \mathrm{capillary} \mathrm{column} 20 \mathrm{~m} \times 0.32 \mathrm{~mm}$, coated with PEG $20 \mathrm{M}$. Arrows indicate temporary replacement of PEG by Ucon HB column. For details see "A typical example".

Substances: Solvent: hexane, one: 5-nonanone, 01:1-octanol, ar: naphthalene, 13-15: n-tridecane-n-pentadecane.

A freshly purchased (DEGUSSA, Hanau, GFR) Pt/Ir capillary, $0.15-0.30 \mathrm{~mm}$ was coiled to a loop diameter of approximately $3 \mathrm{~cm}$. The coil was heated in a flame to a yellow glow while $2 \mathrm{~cm}^{3}$ of oxygen per min were flowing through it. Pieces of glass capillary $2 \mathrm{~cm}$ long were fused on to the ends of the coil. One end was connected, with PTFE shrink tubing, to the exit of a $20 \mathrm{~m} \times 0.32 \mathrm{~mm}$ glass capillary column coated with Ucon HB 5100, the other end to a piece of deactivated glass capillary leading to the flame tip of the FID (Fig. 1). A He flow of $1.5 \mathrm{~cm}^{3}$ $\min ^{-1}$ was applied to the system and the oven was set to $100^{\circ} \mathrm{C}$.

Chromatograms of our adsorption/polarity test mixture were recorded every hour. They showed a slow change from almost total adsorption - i.e. almost no peaks appearing - to increasingly typical results. As expected, first the alkanes, then naphthalene, and finally nonanone and octanol developed reasonable peak shapes. After keeping the system overnight at $220^{\circ} \mathrm{C}$ the chromatograms were identical to those obtained from the same column connected directly the FID.

The column was now replaced by a PEG $20 \mathrm{M}$ column of similar geometry and film thickness. (Both columns were perfectly conditioned and showed very low bleeding at their upper temperature limit). Again, no difference between chromatograms obtained with and without $\mathrm{Pt}$ capillary were obtained. During the night the system was heated to $240^{\circ}$. The next day no change was observed. During a second night the oven temp. was $240^{\circ}$. On the second morning, chromatogram A in Fig. 2 was recorded, showing intense tailing of most peaks. However, the chromatogram differed markedly from the well known results obtained from active columns, insofar as the alkanes showed the most intense peak distortion. The octanol peak was less affected, whereas naph thalene was still eluted with reasonable symmetry. During this day the system was kept at $100^{\circ}$. Periodically recorded chromatograms remained identical to chromatogram A. For the night, the PEG column was replaced by the Ucon $\mathrm{HB}$ column, and the oven was set to $220^{\circ}$. The next morning, again, the PEG column was mounted between vaporizer and Pt capillary. After one hour conditioning at $100^{\circ}$ this system produced the perfect chromatogram $\mathrm{B}$, which is identical to those obtained without a Pt surface. Immediately after this run the oven temperature was increased to $240^{\circ}$. After 4 hours the temperature was lowered to $100^{\circ}$ and chromatogram $\mathrm{C}$ was recorded, showing, with increased intensity, the same trend as chromatogram A. During night the Ucon HB column was again fitted into the system and heated to $220^{\circ}$. The next morning, the PEG column, shortly after replacing the Ucon column, produced chromatogram D. Thus, the adverse effect of the PEG effluents had been corrected. However, after heating to $240^{\circ}$ for two hours only, chromatogram $E$ revealed a very active Pt surface.

The conditioning temperatures applied in this experiment correspond to the temperatures to which the respective columns are heated for short periods during programmed temperature work, or for days during isothermal work. They also correspond to the temperature to which GC/MS interfaces are heated.

\section{Conclusions}

Our work is far from being complete. On the other hand, we consider the reported observations as sufficiently important to be transferred to practical analysts. This paper, therefore, is intended as a recommendation not to use noble metal tubings without having studied their long term behaviour under the proposed conditions. In addi- 
tion to the design of GC/MS interfaces, this recommendation especially applies to the transfer lines vaporizer/ column and column/detector. We would like to emphasize that the difficulties leading to our observations are totally eliminated when well inactivated glass instead of metal is used.

\section{Acknowledgement}

This work was generously sponsored by F. J. Burrus \& Cie, Boncourt, Switzerland. I am indepted to Dr. Joachim Schmid for contributing a confirming GC/MS test, as well as to Dr. Joan Davis for correcting the English.

\section{Literature}

[1] N. Neuner-Jehle, F. Etzweiler, G. Zarske, Chromatographia 6, 211 (1973).

[2] F. Etzweiler, N. Neuner-Jehie, Chromatographia 6, 503 (1973).

[3] K. Grob, H. Jaeggi, Anal. Chem. 45, 1788 (1973). 